\title{
Collection Bernard et Bertrand Bottet de Pierre
} AMROUCHE

\section{Gilles Bounoure}

\section{OpenEdition}

\section{Journals}

\section{Édition électronique}

URL : http://journals.openedition.org/jso/6872

DOI : $10.4000 /$ jso.6872

ISSN : $1760-7256$

Éditeur

Société des océanistes

Édition imprimée

Date de publication : 15 octobre 2013

Pagination : 260-262

ISBN : 978-2-85430-035-2

ISSN : 0300-953x

Référence électronique

Gilles Bounoure, "Collection Bernard et Bertrand Bottet de Pierre Amrouche », Journal de la Société des Océanistes [En ligne], 136-137 | 2013, mis en ligne le 20 octobre 2013, consulté le 10 décembre 2020. URL : http://journals.openedition.org/jso/6872 ; DOI : https://doi.org/10.4000/jso.6872

Ce document a été généré automatiquement le 10 décembre 2020.

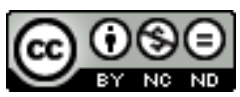

Journal de la société des océanistes est mis à disposition selon les termes de la Licence Creative Commons Attribution - Pas d'Utilisation Commerciale - Pas de Modification 4.0 International. 


\title{
Collection Bernard et Bertrand Bottet de Pierre AMROUCHE
}

\author{
Gilles Bounoure
}

\section{RÉFÉRENCE}

AMROUCHE Pierre, 2013. Collection Bernard et Bertrand Bottet, Paris, Pierre Amrouche

Expertises, 280 p., très nombreuses illustrations.

1 Qu'elle s'occupe d'objets venus d'Océanie ou d'ailleurs, l'« histoire des collections » ne saurait se priver des données livrées par l'étude du collectionnisme, du marché de l'art et des ventes publiques. Une démonstration sans appel en a été récemment apportée par la somme admirable d'Adrienne L. Kaeppler (2011, voir JSO 134) sur la constitution et la dispersion du Leverian Museum, mais les spécialistes connaissent également l'utilité de catalogues comme celui qu'a consacré Steven Hooper aux objets réunis par son grand-père (Phelps, 1976) ou d'enquêtes sur les marchands d'art comparables à celle d'Hermione Waterfield et J. C. H. King (2006), pour s'en tenir à des exemples du meilleur rang. L'apport documentaire du volume dû à Pierre Amrouche (depuis longtemps expert de premier plan dans sa spécialité, actuellement consultant international auprès de Christie's), est d'autant plus précieux qu'il offre aux chercheurs et au public des informations la plupart du temps vouées à se perdre, ou à ne se transmettre que de la façon la plus confidentielle et la moins vérifiable.

Épris d'“ antiquités " au sens large donné à ce terme depuis la Renaissance, Bernard Bottet (1900-1971) et son fils Bertrand (1924-1994) les ont collectionnées pendant un bon demi-siècle, et semblent n'en avoir fait commerce qu'après leur installation à Nice, au sortir de la Deuxième Guerre mondiale, apparemment moins en véritables marchands qu'en amateurs désireux d'améliorer leurs connaissances et leurs sélections d'objets. Cousin de l'abbé Breuil, Bernard Bottet s'était très jeune pris de passion pour les vestiges préhistoriques, qu'il commença à chercher dans la basse vallée de l'Oise, aux gravières très riches, avant de découvrir dans les gorges du Verdon un abri sous 
roche qu'il fouilla avec son fils entre 1946 et 1956 (trouvailles ensuite recueillies dans le musée de Quinson, organisé par Henry de Lumley en 1989). Tous deux publièrent des communications sur ce sujet et d'autres dans le Bulletin de la Société préhistorique française.

3 La collection qu'ils avaient rassemblée, allant de l'art dit " primitif » (Afrique, Océanie, Amériques) aux antiquités asiatiques, européennes et méditerranéennes, fut dispersée entre 1989 et 2012 au cours de huit ventes publiques, dont les six premières comptèrent P. Amrouche parmi les experts. Les descriptions des objets proposés aux enchères s'appuyaient sur deux inventaires manuscrits dressés par les collectionneurs euxmêmes, l'un par le père au tournant des années 1960 (il y est explicitement question d'« anciens francs ", le " nouveau » fut instauré le $1^{\mathrm{er}}$ janvier 1960), l'autre par le fils au milieu des années 1980. Tous deux comportent des dessins et s'accompagnent de précisions variées, lieu et prix d'achat dans le premier, dimensions dans le second, parfois commentaires et éléments de comparaison, faisant de ces inventaires non plus de simples " livres de raison ", mais des sources de premier intérêt sur la circulation des objets d'art « primitif » en France pendant une bonne partie du xxe siècle.

4 Tels sont les documents formant l'essentiel du volume présenté, constitué et édité par P. Amrouche, deux cahiers "format écolier " d'une centaine de pages chacun, complétés de nombreuses feuilles volantes pour le premier inventaire (« Fac-similé des cahiers de Bernard Bottet ", pp. 18-216), et la reproduction en format réduit des pages $\mathrm{du}$ second concernant les seules antiquités océaniennes, américaines et africaines ("Inventaire récapitulatif de Bertrand Bottet », pp. 217-243). La fin de l'ouvrage est consacrée à la dispersion de cette collection ("Les ventes Bottet», pp. 244-278), illustrée par la totalité des objets reproduits au catalogue de chacune de ces ventes. D'autres photos de ces objets, insérées entre les pages en fac-similé, viennent souligner la précision des dessins accompagnant les inventaires. Les textes de présentation sont suivis de leur traduction anglaise, et l'ensemble est édité avec un soin et un raffinement remarquables.

Quant à l'intérêt de ces cahiers, il va bien au-delà de la " valorisation » attendue, du point de vue marchand mais aussi muséographique, de la documentation et de la publication des objets et de leur " provenance » (ou « pedigree »). Exemple presque pris au hasard, il y est fait mention pour quatre spécimens (pp. 61-62) de la " collection Merleau-Ponty » et pour deux autres (p. 55) de la " collection Nelli ", tous évidemment acquis lors de leur dispersion à Drouot en 1931, accompagnée d'un catalogue de 12 pages (Portier, 1931). Dans son étude historique sur le commerce des objets océaniens en France, S. Cazaumayou (2007 : 116-117, avec erreurs sur la date de l'événement et le nom du second collectionneur) évoque cette vente en ces termes :

" Pour la collection Merleau-Ponty, [divers indices font penser] à une collection rassemblée par un médecin ou un scientifique. L'arrière-petite-fille de ce collectionneur confirma cette hypothèse. Merleau-Ponty avait été envoyé comme médecin au bagne de Nouméa en Nouvelle-Calédonie, dans le dernier quart du XIX siècle.

La collection Nelly [sic] est plus énigmatique car la diversité des aires océaniennes ne permet pas de situer ce collectionneur. Nous trouvons des productions de tout l'arc mélanésien avec une dominante pour les îles Fidji, les Nouvelles-Hébrides (Vanuatu) et la Nouvelle-Calédonie. Mais quelques échantillons africains, américains et archéologiques [...] brouillent toutes les chances d'identifier l'origine de cette collection. " 
6 (1864-1934), père du poète et historien René Nelli (1906-1982), brillant spécialiste du catharisme et de la littérature occitane. En décembre 1911, une vente à Drouot avait dispersé une première partie de ses collections, forte de 228 « Objets d'Art et de Haute Curiosité du Moyen Âge \& de la Renaissance ", émaux de Limoges et ivoires gothiques dont certains sont aujourd'hui au Louvre. Le musée des Antiquités nationales conserve (et vend sous forme de réplique en résine) une belle sculpture d'époque magdalénienne en ivoire de mammouth, figurant un cheval, découverte par Léon Nelli dans la grotte des Espélugues à Lourdes qu'il fouilla entre 1886 et 1889, non sans vifs démêlés avec le clergé local.

7 Architecte (et fils d'architecte sculpteur) à Carcassonne, il était féru aussi bien de préhistoire, d'art " primitif ", de " haute époque ", de littérature occitane (sa riche documentation sur le sujet est consultable aux Archives départementales de l'Aude) que d'histoire locale. Cela lui fit fréquenter assidument le chanoine Léopold Verguet (1817-1914), de longue date personnalité centrale de la "Société des Arts et des Sciences » de Carcassonne, mais aussi auteur de relations de ses mois de mission en Mélanésie (voir JSO 135) et possesseur d'une « collection [constituée] vers 1855, objets tous disparus » (Essertel, 2008 : 175, n. 25, sur le rapport de Roger Boulay), ou plus précisément passés, tout ou partie, dans les collections de Léon Nelli (sans que les archives ou les confidences reproduites à la faveur de récentes expositions ou publications consacrées à René Nelli permettent d'en savoir davantage).

Il aurait été satisfaisant de pouvoir clore cette recension par l'établissement définitif de la provenance des deux " haches de danse » (herminettes cérémonielles de Malekula) que les Bottet tenaient de la collection Nelli. Certes Verguet n'a abordé aucune des îles du Vanuatu, mais ce missionnaire fils de commerçants aisés n'avait pas fait vœu de pauvreté au point de renoncer aux plaisirs de la collection. S'il insista tant auprès de ses supérieurs pour revenir d'Océanie à ses propres frais et sur le bateau de son choix, on peut supposer qu'il avait à y faire passer un certain "supplément de bagages " acquis au cours de ses séjours missionnaires comme de ses diverses escales dans le Pacifique, en Nouvelle-Zélande particulièrement. Faute d'inventaire de cette " collection Verguet » ou d'autres documents, il faut, sur ce cas, se borner à évoquer une provenance possible. Mais pour les spécialistes qui sauront en étudier correctement les données, ce que $\mathrm{P}$. Amrouche a eu le mérite de publier des inventaires et des autres traces de la collection Bottet devra être apprécié aussi bien pour les renseignements fiables qu'offre son volume que pour toutes les pistes qu'il ouvre à la recherche.

\section{BIBLIOGRAPHIE}

CAZAUMAYOU Sophie, 2007. Objets d'Océanie : Regards sur le marché de l'art primitif en France, Paris, L'Harmattan.

Journal de la Société des Océanistes, 136-137| 2013 
ESSERTEL Yannick, 2008. Missionnaires maristes et anthropologie au xix ${ }^{\mathrm{e}}$ siècle : aux sources de l'ethnologie et des collections océaniennes ?, Histoire et Missions chrétiennes 8, Paris, Karthala, pp. 159-184.

KAEPPLER Adrienne L., 2011. Holophusicon. The Leverian Museum. An Eighteenth-Century English Institution of Science, Curiosity and Art, Altenstadt, ZKF Publishers.

PHELPS Steven, 1976. Art and Artefacts of the Pacific, Africa and the Americas, The James Hooper Collection, Londres, Hutchinson.

PORTIER André, 1931. Collection L. Nelli. II. Collection Merleau-Ponty. III. Appartenant à divers amateurs. Océanie, Amérique, Afrique. Objets d'art et d'ethnographie dont la vente aux enchères publiques aura lieu Hôtel Drouot, Salle $N^{\circ} 8$, les mercredi 24 et jeudi 25 juin 1931, à 2 heures. Commissaire-priseur : $M^{e}$ Léon Flagel. Expert : M. André Portier, Paris, Étude Flagel.

WATERFIELD Hermione and J. C. H. KING, 2006. Provenance. Twelve Collectors of Ehnographic Art in England 1760-1990, Genève, Somogy-Musée Barbier-Mueller. 